\title{
More ways with neural networks
}

\author{
Ridding neural networks of some of their limitations seems to have suggested ways not just of \\ accounting for the way that attentiveness can improve perception, but for human frailty as well.
}

Physicists, as eager as ever to believe that even biology is only applied physics, are naturally attracted to the notion that neural networks are a useful model for thinking processes capable of pattern recognition. One reason is that the formal description of networks is nicely mathematical. Another is that networks have already been used to solve other problems. An obvious, indeed an almost exact, analogy is that of the structures called spin-glasses, systems in which randomly distributed spins, magnetic or otherwise, interact with each other with a strength dependent on their random separations.

What could be more like the model of, say, the cortex, in which ON/OFF elements stand for neurons and in which the synaptic interconnections are described by coefficients whose magnitude determines the degree to which one neuron influences any other? Work on the spinglasses was the first clearly to show that there is no unique equilibrium state of such a system but, rather, a large set of configurations, each of which represents a local minimum of the physical free energy.

In spin-glasses, these configurations are those in which the system may be trapped if the temperature is not great enough to allow random processes to discover and then to occupy another energy minimum with lower energy (which is the process of annealing). In neural networks, the same local minima correspond to the built-in patterns of the stimuli the network is capable of recognizing. Put crudely, a randomly chosen stimulus will first excite the network into a new state, which will then relax towards that built-in configuration to the stimulus most closely corresponds. In practice, this means that neural networks can recognize even very distorted versions of their built-in patterns.

But how well do the neural networks represent the real world of cognitive pattern recognition? While nobody expects utter precision from such inherently simple models, there are several respects in which they fail to correspond with everyday experience. All of us, for example, know that we can unconsciously, or even consciously, modulate the ease with which we recognize particular patterns by concentrating our attention on them: by what means do we adjust the coefficients representing synaptic strength in the manner this everyday observation implies? And what is to be said of the alltoo-familiar situation that the cortex, when presented with some arbitrary stimulus, can only tell us that it cannot make sense of what it has perceived that no one built-in pattern seems more appropriate than any other?

Two researchers seem now to have been able to adapt the standard neural network model to these needs, suggesting how it may be possible for a working neural network to adjust its own noise level and thus the ease with which particular patterns may be perceived. As a by-product, they suggest how the same modification may allow neural networks quickly to recognize which stimuli are novel.

Maciej Lewenstein and Andrzej Nowak, respectively a theoretical physicist from the Polish Academy of Sciences institute and a psychologist from the University of Warsaw, take the acknowledged defects of existing neural networks as the starting-point for their new construction (Phys. Rev. Lett. 62, 225; 1989). Much of their argument is stimulated by the Monte Carlo computational techniques applied, for example, to the calculation of the stationary states of spin-glasses: by flipping randomly chosen spins in an initial configuration, it is possible to move systematically towards a nearby local minimum of the free energy. But the argument is, mercifully, not just another exercise in computational simulation.

First, Lewenstein and Nowak seek to distinguish between two kinds of built-in patterns or memories, some of which are more 'strongly' memorized than others. The operational difference is that between deep and shallow wells in a free energy surface, but the authors say it is the kind of categorization that might be found in hierarchically organized memories. How to organize such an arrangement?

The underlying assumption in the analogy between neural network models and the real cortex is that both structures must 'learn' which patterns to 'memorize', which requires that neural networks should discover what pattern of coupling strengths between different pairs of elements will embody a learned pattern in a stable fashion - and that neurons in the cortex should similarly grow appropriate synaptic connections.

What the authors find is that their networks will acquire a number of poorly 'remembered' patterns if equal weights are assigned to them in constructing the coupling constants between pairs of elements and if the well-remembered pat- terns are blessed with larger weights Among other things, they find that the capacity of the network to recognize the poorly remembered states in an input stimulus is not impaired. But the unequal treatment does have the effect of reducing the number of the distinctive patterns the network may embody. In reality, there is no reason why the result (hierarchy) should not be advantageous in a real cortex.

The more striking innovation is that used for allowing the model neural network systematically to adjust its own precision, which is done by building into the equations rules that describe the way in which the noise level of the system is locally determined by the the lability of the network during its dynamic evolution.

The noise in the system must be likened to thermal noise, and determines the likelihood that it will spontaneously migrate from one local minimum to another that is more stable. But under the influence of an external pattern, all the spins will be shaken up (neurons will be switched off and on), whereafter the system will evolve to the remembered pattern most nearly determined by the stimulus.

The rules allowing the analogues of attentiveness to be built into recognition by a neural network are recurrence relations relating the noise at one point in the pattern-seeking process to that at an earlier stage by means of quantities representing the numbers of elements in the network changing their state in the interval.

Those working in the field will no doubt be eager to get their hands on the details. For the rest of us, the qualitative implications may suffice. Thus a neural network in the process of homing onto a remembered pattern will, anthropomorphically speaking, do so with increasing confidence. But a network presented with a stimulus that cannot easily be recognized for what it is will change the states of a great many elements in hunting for a solution, the noise will as a result increase and the network may, in effect, be saying 'don't know'. There is also the possibility that the state of a network faced with a badly chosen stimulus will as a consequence be impelled into a noisy state and then driven by random procesess towards the wrong attracting image state. In short, this adaptation of the standard neural network seems both to account for known psychological effects and for a good deal of human frailty - uncertainty and downright misjudgement.

John Maddox 